INPLASY

PROTOCOL

To cite: Wang et al. Efficacy and Safety of Sodium

Oligomannate in the Treatment of Alzheimer's Disease: A

Systematic Review and Metaanalysis. Inplasy protocol

202140009. doi:

10.37766/inplasy2021.4.0009

Received: 01 April 2021

Published: 02 April 2021

Corresponding author:

Yi Wang

wang19830127@126.com

Author Affiliation:

Mental health center, Jiading

District, Shanghai

Support: Company.

Review Stage at time of this submission: The review has not yet started.

Conflicts of interest:

None declared.

\section{Efficacy and Safety of Sodium Oligomannate in the Treatment of Alzheimer's Disease: A Systematic Review and Meta-analysis}

\author{
Wang, Y1; Cheng, C22; Gao, $\mathrm{H}^{3}$; Ban, C4; Shen, $\mathrm{H}^{5}$.
}

Review question / Objective: To evaluate the efficacy and safety of sodium oligomannate in the treatment of Alzheimer's disease.

Condition being studied: Alzheimer's disease (AD), which is a occult onset and progressive with memory impairment in the early stage, and a chronic neurodegenerative disease first reported by Alois Alzheimer in 1907. It is estimated that there are more than $\mathbf{4 0}$ million AD patients in the world, which has become one of the global public health problems. About $90 \%$ of $A D$ patients have behavioral and psychological systerms of dementia (BPSD), which mainly include hallucinations, delusions, abnormal behavior and mood changes. Effective drugs include cholinesterase inhibitors, NMDA receptor antagonists and so on. Sodium oligomannate (code: GV-971) is the first Chinese original and international new drug for Alzheimer's disease targeting the brain-gut axis. Approved for listing on November 2, 2019 for mild to moderate Alzheimer's disease. However, its efficacy and safety are still unclear.

INPLASY registration number: This protocol was registered with the International Platform of Registered Systematic Review and Meta-Analysis Protocols (INPLASY) on 02 April 2021 and was last updated on 02 April 2021 (registration number INPLASY202140009).

\title{
INTRODUCTION
}

Review question / Objective: To evaluate the efficacy and safety of sodium oligomannate in the treatment of Alzheimer's disease.
Condition being studied: Alzheimer's disease (AD), which is a occult onset and progressive with memory impairment in the early stage, and a chronic neurodegenerative disease first reported 
by Alois Alzheimer in 1907. It is estimated that there are more than 40 million $A D$ patients in the world, which has become one of the global public health problems. About $90 \%$ of AD patients have behavioral and psychological systerms of dementia (BPSD), which mainly include hallucinations, delusions, abnormal behavior and mood changes. Effective drugs include cholinesterase inhibitors, NMDA receptor antagonists and so on. Sodium oligomannate (code: GV-971) is the first Chinese original and international new drug for Alzheimer's disease targeting the brain-gut axis. Approved for listing on November 2, 2019 for mild to moderate Alzheimer's disease. However, its efficacy and safety are still unclear.

\section{METHODS}

Participant or population: Patients diagnosed with $A D$, whose behavioral and psychological systerms of dementia (BPSD) were only caused by $A D$, excluding other psychological disorders (such as schizophrenia and bipolar disorder), and cognitive impairment was only caused by $A D$, excluding other nervous system diseases, such as vascular dementia, Lewy body dementia, Parkinson's dementia, etc. The diagnostic criteria were CCMD-3, ICD-10, DSM-IV or NINCDS.ADRDA. All patients participated in the study voluntarily. There was no gender and age limit.

Intervention: Sodium Oligomannate (GV-971).

\section{Comparator: Placebo or no treatment.}

Study designs to be included: Randomized controlled trial (RCT), single-blind, doubleblind or non-blind; English or Chinese literature.

Eligibility criteria: We will include randomized controlled trials (RCTs) study the effectiveness or safety of sodium oligomannate in the treatment of Alzheimer's disease. And RCTs with outcomes or data were presented in a format which can be extracted for analysis.
Information sources: Nine databases of China National Knowledge Infrastructure(CNKI), WanFang Date, VIP, China Biology Medicine disc(CBM), PubMed, Embase, The Cochrane Library, Chinese and American Clinical Trial Registration Center are searched by network computer.

Main outcome(s): Change from baseline in the cognition function of Alzheimer's disease with ADAS-cog and MMSE.

Quality assessment / Risk of bias analysis: Two review authors will independently evaluate the risk of bias of included studies according to the recommended methods of the Cochrane Handbook, any discrepancies were resolved by discussion. Risk of bias will be assessed as 'low risk', 'high risk' or 'unclear risk' for each included study.

Strategy of data synthesis: The metaanalyses will be performed with Review Manager 5.3 only when there is absence of heterogeneity. A fixed-effect model will be used when there is fewer than three trials available; otherwise a random-effects model will be adopted. We will focus on units of analysis and randomization units. If any significant heterogeneity is observed $\left(I^{2} \geq 50 \%\right.$ or $P<$ 0.1 ), a random-effects model will be used; otherwise, a fixed effects model will be used.

Subgroup analysis: When there is significant heterogeneity, we will conduct a subgroup analysis of the severity of $A D$, the dose of the drug, the course of treatment, the dropout rate, and the race of the participants.

Sensitivity analysis: We observed the change of 12 value by deleting each inclusion study in order to explore the source of heterogeneity and conduct sensitivity analysis.

Language: Chinese or English.

Country(ies) involved: China. 
Keywords: Alzheime's disease; Sodium

O I gomannate; Meta-analysis;

Randomized Controlled Trial.

Contributions of each author:

Author 1 - Yi Wang.

Author 2 - Cheng Cheng.

Author 3 - Hongyan Gao.

Author 4 - Chunxia Ban.

Author 5 - Hongyan Shen. 